\title{
New insights into atopic dermatitis
}

\section{Donald Y.M. Leung, ${ }^{1}$ Mark Boguniewicz, ${ }^{1}$ Michael D. Howell, ${ }^{1}$ Ichiro Nomura, ${ }^{1}$ and Qutayba A. Hamid ${ }^{2}$}

${ }^{1}$ Division of Pediatric Allergy-Immunology, National Jewish Medical and Research Center, Department of Pediatrics, University of Colorado Health Sciences Center, Denver, Colorado, USA. ${ }^{2}$ Meakins-Christie Laboratories, Montreal, Quebec, Canada.

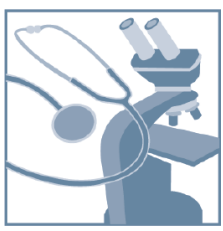

Atopic dermatitis is a chronic inflammatory skin disease associated with cutaneous hyperreactivity to environmental triggers and is often the first step in the atopic march that results in asthma and allergic rhinitis. The clinical phenotype that characterizes atopic dermatitis is the product of interactions between susceptibility genes, the environment, defective skin barrier function, and immunologic responses. This review summarizes recent progress in our understanding of the pathophysiology of atopic dermatitis and the implications for new management strategies.

\section{Historical perspective}

Atopic dermatitis $(\mathrm{AD})$ is a chronic inflammatory skin disease associated with cutaneous hyperreactivity to environmental triggers that are innocuous to normal nonatopic individuals (1). Although written descriptions of $\mathrm{AD}$ date back to the early 1800s, an objective laboratory test does not exist for $\mathrm{AD}$. The diagnosis of $\mathrm{AD}$ is based on the following constellation of clinical findings: pruritus, facial and extensor eczema in infants and children, flexural eczema in adults, and chronicity of the dermatitis.

AD usually presents during early infancy and childhood, but it can persist into or start in adulthood (2). The lifetime prevalence of $\mathrm{AD}$ is $10-20 \%$ in children and $1-3 \%$ in adults. Its prevalence has increased two- to threefold during the past three decades in industrialized countries but remains much lower in countries with predominantly rural or agricultural areas. Wide variations in prevalence have been observed within countries inhabited by groups with similar genetic backgrounds, suggesting that environmental factors play a critical role in determining expression of AD.

A precise understanding of the mechanisms underlying $\mathrm{AD}$ is critical for development of more effective management strategies (Table 1). Various studies indicate that $\mathrm{AD}$ has a complex etiology, with activation of multiple immunologic and inflammatory pathways (3). The clinical phenotype that characterizes AD is the product of complex interactions among susceptibility genes, the host's environment, defects in skin barrier function, and systemic and local immunologic responses. An understanding of the relative role of these factors in the pathogenesis of $\mathrm{AD}$ has been made possible by a variety of approaches, including the analysis of cellular and cytokine gene expression in AD skin lesions in humans as well as gene knockout and transgenic mouse models of candidate genes in AD. The current review will summarize progress in our understanding of the pathophysiology of $\mathrm{AD}$ and implications for therapy.

\section{Atopy as a systemic disease}

Several observations suggest that $\mathrm{AD}$ is the cutaneous manifestation of a systemic disorder that also gives rise to asthma, food allergy, and allergic rhinitis $(1,2)$. These conditions are all characterized by elevated serum IgE levels and peripheral eosinophilia. AD is often

Nonstandard abbreviations used: atopic dermatitis (AD); cutaneous lymphocyteassociated antigen (CLA); Fce receptor I (FcERI); glucocorticoid receptor (GCR); house dust mite (HDM); inflammatory dendritic epidermal cell (IDEC); Langerhans cell (LC); macrophage inflammatory protein (MIP); monocyte chemotactic protein (MCP).

Conflict of interest: D.Y.M. Leung is a consultant, grant recipient, and member of the Speaker's Bureau for Novartis, Fujisawa, and Glaxo/SKB. The remaining authors have declared that no conflict of interest exists.

Citation for this article: J. Clin. Invest. 113:651-657 (2004). doi:10.1172/JCI200421060. the initial step in the so-called "atopic march," which leads to asthma and allergic rhinitis in the majority of afflicted patients. In experimental models of $\mathrm{AD}$, the induction of allergic skin inflammation by epicutaneous application of allergens has been found to augment the systemic allergic response and airway hyperreactivity characteristic of asthma (4).

At least two forms of AD have been delineated: an "extrinsic" form associated with IgE-mediated sensitization involving $70-80 \%$ of the patients, and an "intrinsic" form without IgE-mediated sensitization involving $20-30 \%$ of the patients (5). Both forms of AD have associated eosinophilia. In extrinsic AD, memory $\mathrm{T}$ cells expressing the skin homing receptor, cutaneous lymphocyte-associated antigen (CLA), produce increased levels of Th2 cytokines. These include IL-4 and IL-13, which are known to induce isotype switching to IgE synthesis, as well as IL-5, which plays an important role in eosinophil development and survival. These $\mathrm{CLA}^{+} \mathrm{T}$ cells also produce abnormally low levels of IFN- $\gamma$, a Th 1 cytokine known to inhibit Th2 cell function. Intrinsic AD is associated with less IL-4 and IL-13 production than extrinsic AD.

\section{Immune responses in AD skin}

Clinically unaffected skin in $\mathrm{AD}$ is not normal. It frequently manifests increased dryness and a greater irritant skin response than healthy controls. Unaffected AD skin contains a sparse perivascular T cell infiltrate not seen in normal healthy skin (see Figure 1). Analyses of biopsies from clinically unaffected skin of $\mathrm{AD}$ patients, as compared with normal nonatopic skin, demonstrate an increased number of Th2 cells expressing IL-4 and IL-13, but not IFN- $\gamma$, mRNA (6).

Acute eczematous skin lesions present clinically as intensely pruritic, erythematous papules associated with excoriation and serous exudation. In the dermis of acute lesions, there is a marked infiltra-

\section{Table 1}

Important concepts in the pathobiology of $A D$

Cutaneous hyperreactivity to environmental triggers

Skin barrier dysfunction

Immunologic triggers: food and inhalant allergens, microbial infection, autoantigens

Systemic Th2 response; first step in atopic march

Biphasic T cell response in the skin (Th2 cells in acute AD; Th1 cells in chronic $A D$ )

Reduced skin innate immune response

Skin inflammatory response driven by chemokine expression

Skin remodeling in chronic $A D$

Key effector cells: T cells, FcERI+/lgE+ DCs, keratinocytes 


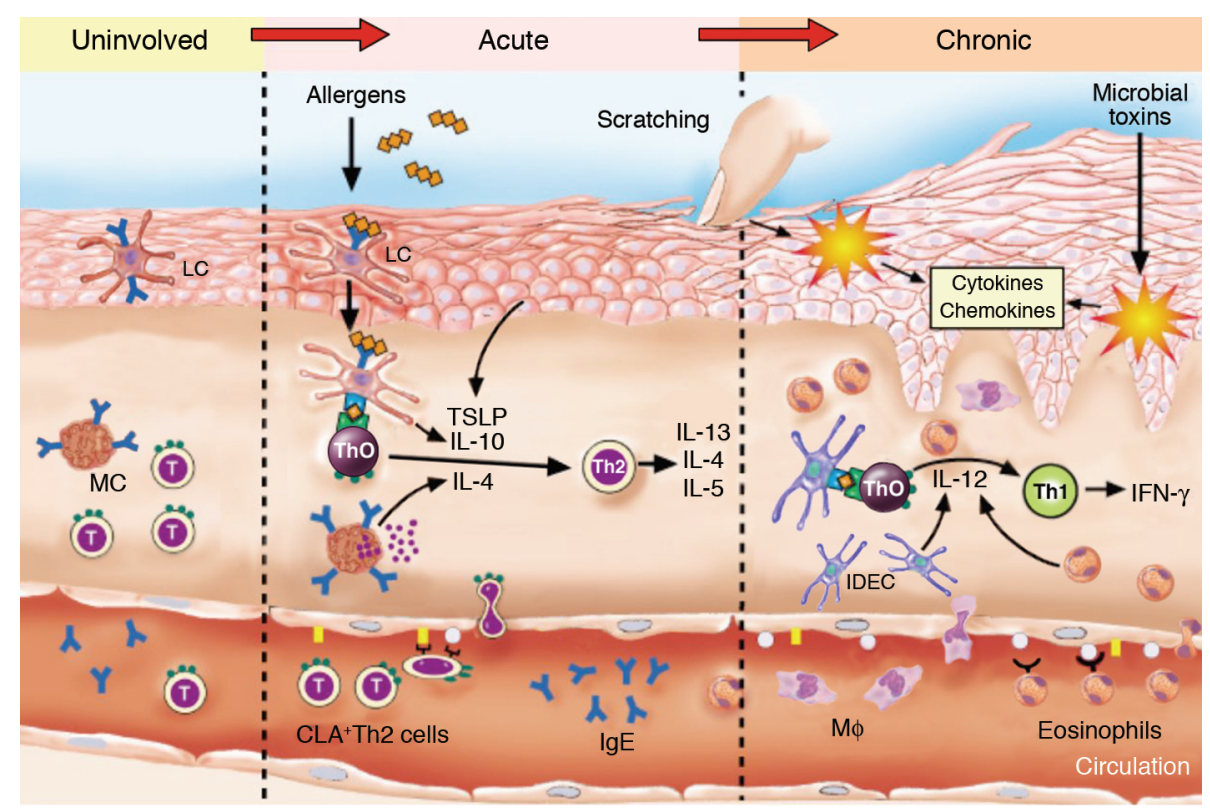

Figure 1

Immunologic pathways in AD. Th2 cells circulating in the peripheral blood of AD patients result in elevated serum IgE and eosinophils. These T cells express the skin homing receptor, CLA, and recirculate through unaffected AD skin where they can engage allergen-triggered $\lg \mathrm{E}^{+} \mathrm{LC}$ s and mast cells (MCs) that contribute to Th2 cell development. Skin injury by environmental allergens, scratching, or microbial toxins activates keratinocytes to release proinflammatory cytokines and chemokines that induce the expression of adhesion molecules on vascular endothelium and facilitate the extravasation of inflammatory cells into the skin. Keratinocyte-derived thymic stromal lymphopoietin (TSLP) and DC-derived IL-10 also enhance Th2 cell differentiation. AD inflammation is associated with increased Th2 cells in acute skin lesions, but chronic AD results in the infiltration of inflammatory IDECs, macrophages $(\mathrm{M} \phi)$, and eosinophils. IL-12 production by these various cell types results in the switch to a Th1-type cytokine milieu associated with increased IFN- $\gamma$ expression. Figure modified with permission from The Journal of Allergy and Clinical Immunology (35).

tion of $\mathrm{CD}^{+}$activated memory $\mathrm{T}$ cells. When compared to normal skin or uninvolved skin of $\mathrm{AD}$ patients, acute skin lesions have a significantly greater number of IL-4, IL-5, and IL-13 mRNA-expressing cells, but few IFN- $\gamma$ or IL-12 mRNA-expressing cells. APCs (e.g., Langerhans cells [LCs], inflammatory dendritic epidermal cells [IDECs], and macrophages) in lesional and, to a lesser extent, in nonlesional skin bear IgE molecules (7).

Chronic lichenified skin lesions have undergone tissue remodeling (Figure 2) due to chronic inflammation and are characterized by thickened plaques with increased markings (lichenification) and dry, fibrotic papules. There is an increased number of IgE-bearing LCs and IDECs in the epidermis, and macrophages dominate the dermal mononuclear cell infiltrate. Eosinophils also contribute to the inflammatory response, and $\mathrm{T}$ cells remain present, although in smaller numbers than seen in acute $\mathrm{AD}$. Chronic $\mathrm{AD}$ skin lesions have significantly fewer IL-4 and IL-13 mRNA-expressing cells, but greater numbers of IL-5, GM-CSF, IL-12, and IFN- $\gamma$ mRNA-expressing cells, than in acute AD. Recent studies suggest that collagen deposition during chronic $\mathrm{AD}$ is due to increased gene expression of the profibrotic cytokine, IL-11 (8).

The evolution of AD skin lesions is orchestrated by the local tissue expression of proinflammatory cytokines and chemokines. Cytokines such as TNF- $\alpha$ and IL-1 from resident cells (keratinocytes, mast cells, and DCs) bind to receptors on vascular endothelium, activating cellular signaling including the NF- $\mathrm{KB}$ pathway and inducing expression of vascular endothelial cell adhesion molecules. These events initiate the process of tethering, activation, and adhesion to the endothelium followed by extravasation of inflammatory cells. Once the inflammatory cells have infiltrated into the tissue, they respond to chemotactic gradients established by chemoattractant cytokines and chemokines, which emanate from sites of injury or infection (9). These molecules play a central role in defining the nature of the inflammatory infiltrate in AD (10). IL-16, an LC-derived chemoattractant cytokine for $\mathrm{CD}^{+} \mathrm{T}$ cells, is increased in acute $\mathrm{AD}$ skin lesions. C-C chemokine ligand 27 is highly upregulated in $\mathrm{AD}$ and preferentially attracts $\mathrm{CLA}^{+} \mathrm{T}$ cells into the skin. As compared to psoriasis, the $\mathrm{C}-\mathrm{C}$ chemokines, RANTES, monocyte chemotactic protein-4, and eotaxin (Figure 3) are increased in $\mathrm{AD}$ skin lesions and likely contribute to the chemotaxis of C-C chemokine receptor 3-expressing (CCR3-expressing) eosinophils, macrophages, and Th2 lymphocytes into $\mathrm{AD}$ skin. Selective recruitment of CCR4-expressing Th2 cells into $\mathrm{AD}$ skin may also be mediated by macrophage-derived chemokine and thymus and activation-regulated cytokine, which are increased in AD. Persistent skin inflammation in chronic lesions may be due to elevated IL-5 and GM-CSF expression in the skin leading to enhanced survival of eosinophils and monocyte-macrophages as well as LCs. In addition, extracellular matrix molecules deposited into chronic skin lesions have been found to enhance the survival of memory T cells (11).

\section{Key effector cells in AD skin}

T cells. The key role of immune effector T cells in AD is supported by the observation that individuals with primary $\mathrm{T}$ cell immunodeficiency disorders frequently have elevated serum IgE levels and eczematous skin lesions which clear following successful bone marrow transplantation. Furthermore, in animal models of AD, the eczematous rash does not occur in the absence of T cells. Use of the atopy patch test technique as a model for the induction of eczema in patients with $\mathrm{AD}$ has shown that house dust mite (HDM) allergen-induced skin lesions display two phases: an initial phase with predominantly IL-4 producing Th2 cells and a subsequent phase after 24 to 48 hours characterized by IFN- $\gamma$ producing Th1 cells (1). This switch is thought to be initiated by the local production of IL-12 from infiltrating eosinophils and/or IDECs (3). Activated T cells expressing Fas ligand have also been shown to induce keratinocyte apoptosis contributing to the spongiosis found in acute $\mathrm{AD}(12)$. This process is mediated by IFN- $\gamma$, which upregulates Fas on keratinocytes.

The important role that Th1 and Th2 cytokines play in the skin inflammatory response has been demonstrated in experimental models of allergen-induced allergic skin inflammation in mice with 

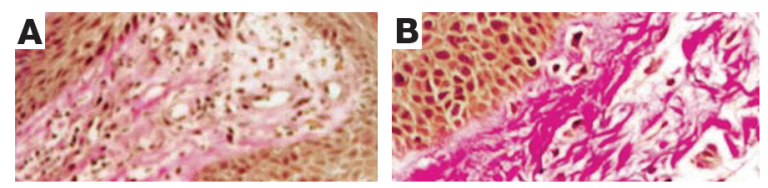

Figure 2

Skin remodeling in AD. Van Gieson staining (original magnification, $\times 400$ ) from acute $A D(A)$ and chronic $A D(B)$ showing extensive fibrosis in chronic, as compared to acute, AD lesions. Figures reproduced with permission from The Journal of Allergy and Clinical Immunology (8).

targeted deletions or overexpression of these cytokines. In this regard, transgenic mice genetically engineered to overexpress IL-4 in their skin develop inflammatory pruritic skin lesions similar to $\mathrm{AD}$, suggesting that local skin expression of Th2 cytokines plays a critical role in $\mathrm{AD}$ (13). Allergen-sensitized skin from IL-5 knockout mice has been found to have no detectable eosinophils and exhibits decreased thickening; skin from IL-4 knockout mice displays normal thickening of the skin layers, but has a reduction in eosinophils, while skin of IFN- $\gamma$ knockout mice is characterized by reduced dermal thickening (14).

Antigen-presenting cells. AD skin, as compared to nonatopic skin, contains an increased number of IgE-bearing LCs and IDECs expressing the high affinity IgE receptor Fce receptor I (FcERI) (7). The increased expression of FcERI on DCs in atopic skin is due to enhanced expression of the FceRI $\gamma$ chain and is preserved by increased IgE levels (15). LCs and IDECs play an important role in allergen presentation to Th2 and Th1 cells, respectively (3). FceRIbound IgE on LCs facilitates the capture and internalization of allergens prior to their processing and antigen presentation to $T$ cells in atopic skin. FceRI ${ }^{+} / \mathrm{IgE}^{+} \mathrm{LCs}$ may also migrate to the lymph nodes and stimulate naive T cells to expand the pool of Th2 cells. The clinical importance of these cells is supported by the observation that, using an experimental model of aeroallergen-induced patch test reactions on atopic skin, the presence of $\mathrm{FccRI}^{+} / \mathrm{IgE}^{+} \mathrm{LC}$ is required to provoke eczematous skin lesions. IL-10 expression by APCs and $\mathrm{T}$ cells has recently been demonstrated to play a key role in Th2 cell development in an animal model of AD (16).

Keratinocytes. Epidermal keratinocytes from AD patients produce a unique profile of chemokines and cytokines following mechanical stimulation, e.g., scratching, or exposure to proinflammatory cytokines, including abnormally high levels of RANTES following stimulation with TNF- $\alpha$ and IFN- $\gamma$. They are also an important source of thymic stromal lymphopoietin, which activates DCs to prime naive Th cells to produce IL-4 and IL-13. These observations may explain the link between scratching and the triggering of Th2-mediated skin inflammation in $\mathrm{AD}$. As discussed below, keratinocytes in $\mathrm{AD}$ are also deficient in their ability to synthesize antimicrobial peptides needed for innate immune responses against microbes $(17,18)$.

\section{Skin barrier dysfunction}

$\mathrm{AD}$ is characterized by dry skin and even involves nonlesional skin and increased transepidermal water loss. This impairment of the skin barrier function in $\mathrm{AD}$ leads to increased antigen absorption contributing to the cutaneous hyperreactivity characteristic of AD. In particular, ceramides serve as the major water-retaining molecules in the extracellular space of the cornified envelope, and the barrier function of these complex structures is provided by a matrix of structural proteins that are bound to ceramides (19). A reduced con- tent of ceramides has been reported in the cornified envelope of both lesional and nonlesional skin in AD patients. The increased susceptibility to irritants in $\mathrm{AD}$ may therefore represent a primary defect of epidermal differentiation compounded by the presence of inflammation-induced skin damage.

\section{Genetics}

$\mathrm{AD}$ is a genetically complex disease that has a high familial occurrence. Twin studies of AD have shown concordance rates of 0.72-0.86 in monozygotic, and $0.21-0.23$ in dizygotic, twin pairs, indicating that genetic factors play an important role in the development of this disease (20). Relevant to AD as part of a systemic atopic disorder, candidate genes involving IgE and Th2 cytokines have been identified. There has been particular focus on chromosome 5q31-33, as it contains a clustered family of Th2 cytokine genes, i.e., IL-3, IL-4, IL-5, IL-13, and GM-CSF (21). Case-control comparisons have suggested a genotypic association between the $\mathrm{T}$ allele of the $-590 \mathrm{C} / \mathrm{T}$ polymorphism of the IL-4 gene promoter region and AD. The fact that this allele is associated with increased IL-4 gene promoter activity suggests that it may increase allergic responses in AD. Similarly, IL-13 coding region variants, a gain-of-function polymorphism in the $\alpha$ subunit of the IL-4 receptor (located on chromosome 16q12), and a functional mutation in the promoter region of RANTES (located on chromosome $17 q 11$ ) have been reported in AD. There have been controversial linkage findings between $\mathrm{AD}$ and markers on chromosome $11 q 13$, including the gene encoding for the $\beta$-chain of the high affinity receptor for $\operatorname{IgE}(F c \varepsilon R I \beta)$. Most of these studies included patients

\section{A}
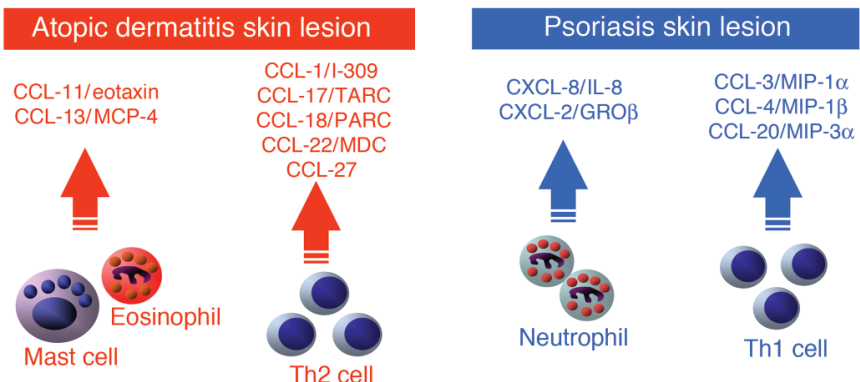

B Skin-homing T cell
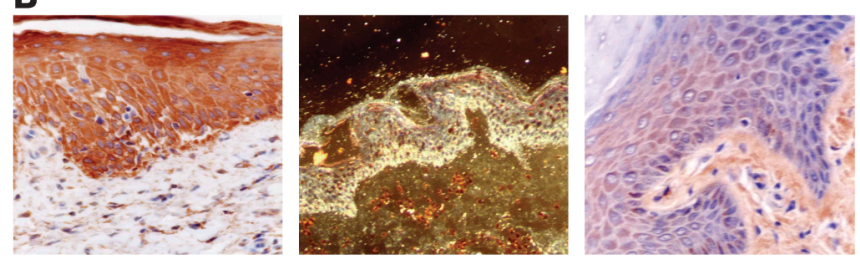

\section{Figure 3}

Role of chemokines in AD. (A) Chemokines activated in AD versus psoriasis. (B) Representative immunostaining for immunochemical staining of a chronic $A D$ skin section using antibody to eotaxin (left panel; original magnification, $\times 400$ ), in situ hybridization of chronic AD skin for MCP-4 using a complementary RNA radio-labeled probe. The image of mRNAcRNA complex was developed with autoradiography. Dark field illustration shows positive signal in the epidermis of inflammatory cells (middle panel; original magnification, $\times 400$ ). Immunostaining of psoriasis skin section with antibody to eotaxin shows weak staining (right panel; original magnification, $\times 400$ ). TARC, thymus and activation-regulated cytokine; PARC, pulmonary and activation-regulated chemokine; GRO $\beta$, growth-related $\beta$. 
with elevated IgE levels, i.e., patients with extrinsic AD. Thus, studies of so-called "pure" or intrinsic AD are needed to identify AD genes unrelated to IgE biology. Importantly, linkages to AD have generally not corresponded to loci associated with asthma, suggesting the presence of separate or additional genes in $\mathrm{AD}$ that may not act through atopic mechanisms.

Genome screens have been performed to identify susceptibility loci for AD. One screen in families of German and Scandinavian children found a linkage for $\mathrm{AD}$ on chromosome $3 \mathrm{q} 21$ (20). This region encodes the costimulatory molecules CD80 and CD86 and therefore may modulate $\mathrm{T}$ cell responses. A second screen reported linkage of $\mathrm{AD}$ to loci on chromosomes $1 \mathrm{q} 21,17 \mathrm{q} 25$, and 20p. Interestingly, these same regions are known to contain psoriasis susceptibility genes, which suggests common candidate genes involved in the control of skin inflammation. Although $\mathrm{AD}$ and psoriasis are distinct skin diseases, both conditions involve dry, scaly skin and disrupted epidermal differentiation.

A Glu420Lys polymorphism variant in the SPINK5 gene, which encodes serine proteinase inhibitor, Kazal type 5, has shown significant association with AD in two independent cohorts (22). This gene is expressed in the outermost layers of the skin and has been implicated in Netherton disease, an autosomal recessive disorder characterized by ichthyosis and atopy. It may have a protective role against allergens that are serine proteinases.

The rapid rise in prevalence of atopic diseases has been ascribed to the so-called hygiene hypothesis. According to this theory, early infections or exposure to microbial-derived material, such as LPS, early in life prevent the development of Th2-driven allergic disease. Thus, polymorphisms of genes involved in the recognition of microbial material may alter the balance between Th1- and Th2-driven immune responses and could change an individual's susceptibility to development of allergic diseases. Indeed, polymorphisms of a number of innate immunity genes such as CD14 and Toll-like receptors have been associated with the development of allergy. Recently, a polymorphism (G2722C) that results in functional impairment of caspase recruitment domain-containing protein 15 , an intracellular receptor for LPS involved in NF- $\kappa \mathrm{B}$ activation, has been associated with a twofold increased risk for development of AD (23). Thus, not only reduced microbial exposure in the environment, but also impaired molecular recognition of microbial molecules, may give rise to enhanced Th2 responses.

\section{Factors contributing to flares of AD}

General. The skin represents the interface between the body and the surrounding environment. It is therefore subjected to numerous insults, which act as triggers of inflammation. These include irritants as well as allergens. These factors trigger the scratching that ensues to induce and sustain the inflammatory cascade initiated by release of proinflammatory cytokines from atopic keratinocytes. Stress itself has been found to induce immunologic changes and combined with scratching will trigger the exacerbation of AD.

Allergens. Placebo-controlled, food challenge studies have demonstrated that food allergens can induce eczematoid skin rashes in nearly $40 \%$ of children with moderate to severe $\mathrm{AD}$. In a subset of these patients, urticarial reactions, or noncutaneous symptoms, are elicited, which can trigger the itch-scratch cycle that flares this skin condition. Children with food allergies generally have positive immediate skin tests or serum IgE directed to various foods, particularly eggs, milk, wheat, soy, and peanuts. Importantly, food allergen-specific $\mathrm{T}$ cells have been cloned from the skin lesions of patients with $\mathrm{AD}$, pro- viding direct evidence that foods can contribute to skin inflammation. In mouse models of $\mathrm{AD}$, oral sensitization with foods results in the elicitation of eczematous skin lesions on repeat oral food challenges.

After the age of 3, children frequently outgrow food allergy but may become sensitized to inhalant allergens. Pruritus and skin lesions can develop after intranasal or bronchial inhalation challenge with aeroallergens in sensitized AD patients. Epicutaneous application of aeroallergens (e.g., HDMs, weeds, animal danders, and molds) by atopy patch test on uninvolved skin of AD patients elicits eczematoid reactions in 30-50\% of patients with AD. A combination of effective HDM-reduction measures has been reported to improve $\mathrm{AD}$. The degree of IgE sensitization to aeroallergens is directly associated with the severity of $\mathrm{AD}$. The isolation from AD skin lesions and allergen patch test sites of T cells that selectively respond to Dermatophagoides pteronyssinus and other aeroallergens supports the concept that immune responses in AD skin can be elicited by environmental aeroallergens.

Autoallergens. Patients with severe AD have been reported to generate $\operatorname{IgE}$ antibodies directed against human proteins. The autoallergens identified to date have been intracellular proteins, which can be detected in IgE immune complexes of AD sera. These data suggest that while IgE immune responses are initiated by environmental allergens, allergic inflammation can be maintained by the release of human proteins derived from damaged skin in chronic AD.

Microbes. Most patients with $\mathrm{AD}$ are colonized with Staphylococcus aureus and suffer relapses of their skin disease following overgrowth of this organism (24). The importance of S. aureus is supported by the observation that, in $\mathrm{AD}$ patients with secondary infection, treatment with a combination of antistaphylococcal antibiotics and topical corticosteroids results in greater clinical improvement than treatment with topical corticosteroids alone. One strategy by which $S$. aureus exacerbates $\mathrm{AD}$ is by secreting toxins called superantigens, which stimulate activation of $\mathrm{T}$ cells and macrophages. Most AD patients make specific IgE antibodies directed against staphylococcal superantigens (25), and these IgE antisuperantigens correlate with skin disease severity. Superantigens also induce corticosteroid resistance, suggesting that several mechanisms exist by which superantigens increase AD severity.

Increased binding of $S$. aureus to skin is driven by underlying $\mathrm{AD}$ skin inflammation. This is clinically supported by studies demonstrating that treatment with topical corticosteroids or tacrolimus reduces $S$. aureus counts on atopic skin. In experimental animal models, $S$. aureus binding was significantly greater at skin sites with Th2as compared to Th1-mediated skin inflammation due to IL-4induced expression of fibronectin. AD skin has also been found to be deficient in antimicrobial peptides needed for host defense against bacteria, fungi, and viruses $(17,18)$. Thus, once $S$. aureus binds to AD skin, inadequate host defense allows bacteria to colonize and grow. The lack of skin innate immune responses may predispose these patients to infection as well to fungi and viruses. Patients with AD have an increased propensity toward disseminated infections with herpes simplex or vaccinia virus. As such, smallpox vaccination is contraindicated in patients with $\mathrm{AD}$ unless there is imminent danger of exposure to smallpox.

\section{Translating the lessons of molecular immunology into current management of AD}

Successful management of AD requires a multipronged approach. This includes the avoidance of irritants and specific immunologic stimuli, including foods and aeroallergens, which can either induce 
the dermatitis or trigger the itch-scratch cycle that results in AD (1). Skin hydration and use of emollients to repair the impaired skin barrier function is a key part of management. Addition of a "ceramidedominant" emollient to standard therapy results in both clinical improvement and decreased transepidermal water loss and improvement of stratum corneum integrity in children with "stubborn-torecalcitrant” $\mathrm{AD}$ (26). Although viral or fungal infection can trigger $\mathrm{AD}, S$. aureus colonization or infection is the most common cause of increased AD severity. In such patients a course of antibiotics, in combination with anti-inflammatory therapy, will lead to better control of skin disease. The key to successful long-term management of $\mathrm{AD}$ is the introduction of effective anti-inflammatory therapy.

Topical corticosteroids. This class of drugs is the mainstay of antiinflammatory treatment, showing efficacy in the control of both acute and chronic skin inflammation. Corticosteroids mediate their anti-inflammatory effects through a cytoplasmic glucocorticoid receptor (GCR) in target cells. Upon ligand binding, the corticosteroid/GCR complex translocates into the nucleus where it mediates its anti-inflammatory effects via two major actions. First, the GCR complex can induce gene transcription by binding of GCR dimers to glucocorticoid response elements (a process known as transactivation) in the promoter regions of target genes. Transactivation is mainly responsible for the unwanted side effects of glucocorticoids. The major mechanism by which GCR mediates its anti-inflammatory effects is via a process called transrepression, which is independent of GCR DNA binding. In this process, ligand-bound GCR binds to various transcription factors, including activator protein- 1 and NF- $\kappa B$, via protein-protein interactions to inhibit the transcriptional activity of various proinflammatory genes encoding proinflammatory proteins such as cytokines (IL-1, IL-2, IL-3, IL-4, IL-5, IL-6, IL-11, IL-13, TNF- $\alpha$, and GM-CSF), chemokines (IL-8, RANTES, macrophage inflammatory protein- $1 \alpha$ [MIP- $1 \alpha]$, monocyte chemotactic protein1 [MCP-1], MCP-3, MCP-4, and eotaxin), and adhesion molecules (ICAM-1,VCAM-1, and E-selectin).

Due to concerns about potential side effects associated with chronic use, topical corticosteroids have not been used for maintenance therapy, especially on nonlesional skin, in AD. However, normal-appearing skin in AD is associated with subclinical inflammation, suggesting that maintenance anti-inflammatory therapy may be required to prevent relapse. Indeed, several studies with fluticasone propionate have shown that once control of clinical disease is achieved, long-term control can be maintained with twice weekly therapy (27).

Topical calcineurin inhibitors. Topical FK506 and pimecrolimus have recently been FDA-approved for the treatment of AD. Both agents act by binding with high affinity to the $12 \mathrm{kDa}$ macrophilin and inhibit the phosphatase activity of the calcium-dependent serine/threonine phosphatase, calcineurin. In the presence of these calcineurin inhibitors, the transcription factor, nuclear factor of activated $\mathrm{T}$ cell protein (NF-ATp), is not dephosphorylated and therefore cannot translocate into the nucleus to activate transcription of various Th1 and Th2 cytokine genes. Tacrolimus and pimecrolimus inhibit the activation of a number of key effector cells involved in $\mathrm{AD}$, including T cells and mast cells.

Short-term, multicenter, blinded, vehicle-controlled trials, in both adults and children, have shown both topical tacrolimus and pimecrolimus to be effective. In a minority of patients, stinging and local irritation have been reported with this new class of drugs, but overall they have been found to be quite safe. Long-term studies with both drugs have been performed in adults and children, with demonstrated sustained efficacy and no significant side effects. Unlike topical glucocorticoids, topical calcineurin inhibitors are not atrophogenic and have been used safely for facial and eyelid eczema. Several studies with pimecrolimus cream have also found that introducing the medication at the earliest signs of clinical disease results in significantly less need for corticosteroid "rescue" therapy.

The approval of topical calcineurin inhibitors for the treatment of AD represents a significant advance in our management options for this disease. The distinction between pimecrolimus and tacrolimus is that pimecrolimus is a cream that is somewhat weaker than tacrolimus but less irritating. Tacrolimus is currently marketed as an ointment that is more potent but also more irritating. Importantly, there are situations in which topical calcineurin inhibitors may be advantageous over topical corticosteroids and may be useful as firstline therapy. These would include treatment of patients who are poorly responsive to topical steroids or have steroid phobia, and treatment of face and neck dermatitis where ineffective, low-potency topical corticosteroids are usually used due to fears of steroid-induced skin atrophy. The potential use of topical calcineurin inhibitors as maintenance therapy is also intriguing for prevention of $\mathrm{AD}$ flares and progression of the atopic march $(28,29)$. However, although systemic absorption of these compounds is low, there is a need for careful surveillance to rule out the possibility that skin cancers and increased viral skin infections will appear when such agents are used long-term.

Alternative anti-inflammatory approaches. UV light therapy can be a useful treatment modality for chronic recalcitrant AD. The photoimmunologic effects target key cells in atopic inflammation, such as LCs and keratinocytes, interfering with cytokine production and decreasing the expression of activation markers such as HLA-DR and IL-2 receptor on CLA ${ }^{+} \mathrm{T}$ cells. Unlike traditional UVA-UVB phototherapy, which appears less effective for acute exacerbations and acts primarily in the epidermis, high-dose UVA1 therapy has

\section{Table 2}

Evolving therapeutic targets in $\mathrm{AD}$

\section{Anti-allergic approaches Anti-lg $\mathrm{E}$ Allergen-selective immunotherapy Immunization with $\mathrm{CpG}$ motifs \\ T cell targets}

Probiotics (e.g., Lactobacillus rhamnosus strain GG)

Reduction of Th2 cytokine responses (anti-IL-4, soluble IL-4 receptor, anti-IL-13, antisense oligonucleotide approaches)

Mycobacterium vaccae vaccination

Inhibition of T cell activation; e.g., alefacept (blocks T cell CD2-leukocyte functional antigen-1 [LFA-1] and -LFA-3 APC interactions), efalizumab (blocks LFA-1-ICAM-1 and -ICAM-2 APC interactions)

Anti-inflammatory agents Oral pimecrolimus TNF inhibitors

Antimicrobial approaches augmenting $\mathrm{T}$ cell responses Antimicrobial peptides

Blockade of inflammatory cell recruitment

Chemokine antagonists ( $\mathrm{C}-\mathrm{C}$ chemokine receptor-4, cutaneous lymphocyte-associated antigen)

CLA inhibitors

CpG, cytosine phosphate guanine. 
been shown to significantly decrease dermal IgE-binding cells, including mast cells, LCs, and DCs. UVA1 also inhibits LC migration out of the epidermis.

Cyclosporin A is a potent systemic calcineurin inhibitor. A number of studies have demonstrated its efficacy in both children and adults with severe, refractory $\mathrm{AD}$, although toxicity, primarily renal, limits its chronic use. Preliminary data with oral pimecrolimus suggest that it may have a greater safety margin than either systemic cyclosporin or tacrolimus, which could make treatment more acceptable to some patients and reach inflammatory targets not accessible to topical medications (30). Antimetabolites, including mycophenolate mofetil, a purine biosynthesis inhibitor, methotrexate, and azathiaprine, have also been utilized for recalcitrant $\mathrm{AD}$, although the potential for systemic toxicities restricts their use and requires close monitoring.

\section{New approaches to modulate atopic inflammation}

Although a number of anecdotal and case reports suggest clinical benefit from allergen-specific desensitization in $\mathrm{AD}$, double-blinded, controlled trials have failed to show consistent efficacy of immunotherapy compared to placebo in the treatment of AD. Recently, omalizumab, a humanized IgG1 monoclonal antibody against IgE that recognizes and masks an epitope in the $\mathrm{CH} 3$ region of $\mathrm{IgE}$ responsible for binding to the high-affinity FceR on mast cells and basophils, has been shown to be effective in treatment of allergic asthma and allergic rhinitis. Thus, it could potentially neutralize the effects of IgE in $\mathrm{AD}$. However, the high serum IgE levels seen in $\mathrm{AD}$ may limit the usefulness of this antibody, although it may have a role in food-induced AD. In this respect, treatment of a population of peanut-allergic patients with anti-IgE significantly increased their threshold of sensitivity to peanuts on oral food challenge, suggesting protection against unintended ingestion of the food allergen (31).

Insights into the role that certain cells and cytokines play in $\mathrm{AD}$ create opportunities for the development of targeted therapy. However, given the complexity of the biological processes involved, none of the tested compounds to date has proven to be the magic bullet. Some promising agents have been limited by associated toxicity. In this respect, several studies with recombinant human IFN- $\gamma$ demonstrated clinical efficacy that correlated with a decrease in blood eosinophilia, even with long-term therapy. Early treatment with microbial probiotics may be more beneficial by boosting Th1 immune responses in $\mathrm{AD}$ (32). Additional approaches include cytokine modulation (e.g., soluble IL-4 receptor; anti-IL-5 monoclonal antibody; TNF inhibitors), blockade of inflammatory cell recruitment (chemokine receptor antagonists, CLA inhibitors, ref. 33), inhibition of T cell activation (alefacept, efalizumab, ref. 34), and use of synthetic antimicrobial peptides (Table 2).

\section{Future directions}

Our challenge for the future will be the development of more effective and safer drugs in the treatment of AD. Given the complexity of immune pathways that lead to $\mathrm{AD}$, it is possible that more selective anti-inflammatory or immunomodulatory agents will be less effective. Thus, it will be important to better characterize the key immune pathways leading to the different phenotypes of $\mathrm{AD}$, as medications may vary in their effectiveness for treatment of different forms of $\mathrm{AD}$. For example, it is important to resolve whether the immune pathways leading to so-called intrinsic (or pure) vs. extrinsic (IgEmediated) AD are fundamentally different. The role that IgE plays in allergic disease is still debated. Animal models of allergy can occur in IgE-knockout mice. This molecule may primarily act to facilitate allergen processing for Th2 cell activation and immediate reaction at extremely low environmental concentrations of allergens. Immune responses to allergen may occur in the absence of $\mathrm{IgE}$ in intrinsic $\mathrm{AD}$ but require higher allergen concentrations for $\mathrm{T}$ cell activation in the absence of immediate reaction. Indeed, atopy patch tests can be positive in a subset of AD patients lacking IgE responses to the relevant allergen. The relative role of microbes and autoantigens in the initiation and progression of $\mathrm{AD}$ also requires further clarification.

New advances are likely to require better definitions for the various clinical phenotypes of $\mathrm{AD}$, including identification of the susceptibility genes leading to the different forms of AD and delineation of the relative role of immunoregulatory abnormalities and structural skin barrier defects underlying AD skin. New treatment paradigms are needed for preventing disease progression of $\mathrm{AD}$ to more severe forms of this skin disease and halting the so-called atopic march, which results in development of asthma. The factors that determine the chronicity, skin remodeling, and natural history of this disease remain poorly characterized. An understanding of the genes responsible for individual variation in response to therapy will be tied to the development of pharmacogenetics and the targeting of effective therapies to the different phenotypes of AD.

Address correspondence to: Donald Y.M. Leung, National Jewish Medical and Research Center, 1400 Jackson Street, Room K926, Denver, Colorado 80206, USA. Phone: (303) 398-1379; Fax: (303) 270-2182; E-mail: leungd@njc.org.
1. Leung, D.Y., and Bieber, T. 2003. Atopic dermatitis. Lancet. 361:151-160.

2. Spergel, J.M., and Paller, A.S. 2003. Atopic dermatitis and the atopic march. J. Allergy Clin. Immunol. 112:S128-S139.

3. Novak, N., Bieber, T., and Leung, D.Y.M. 2003. Immune mechanisms leading to atopic dermatitis. J. Allergy Clin. Immunol. 112:S128-S139.

4. Spergel, J.M., et al. 1998. Epicutaneous sensitization with protein antigen induces localized allergic dermatitis and hyperresponsiveness to methacholine after single exposure to aerosolized antigen in mice. J. Clin. Invest. 101:1614-1622.

5. Novak, N., and Bieber, T. 2003. Allergic and nonallergic forms of atopic diseases. J. Allergy Clin. Immunol. 112:252-262.

6. Hamid, Q., Boguniewicz, M., and Leung, D.Y. 1994. Differential in situ cytokine gene expression in acute versus chronic atopic dermatitis. J. Clin. Invest. 94:870-876.
7. Novak, N., Kraft, S., and Bieber, T. 2003. Unraveling the mission of FcepsilonRI on antigen-presenting cells. J. Allergy Clin. Immunol. 111:38-44.

8. Toda, M., et al. 2003. Polarized in vivo expression of IL-11 and IL-17 between acute and chronic skin lesions. J. Allergy Clin. Immunol. 111:875-881.

9. Ono, S.J., et al. 2003. Chemokines: roles in leukocyte development, trafficking, and effector function. J. Allergy Clin. Immunol. 111:1185-1199.

10. Nomura, I., et al. 2003. Distinct patterns of gene expression in the skin lesions of atopic dermatitis and psoriasis: a gene microarray analysis. J. Allergy Clin. Immunol. 112:1195-1202.

11. Akdis, M., et al. 2003. T helper (Th) 2 predominance in atopic diseases is due to preferential apoptosis of circulating memory/effector Th1 cells. FASEB J. 17:1026-1035.

12. Trautmann, A., et al. 2000. T cell-mediated Fasinduced keratinocyte apoptosis plays a key pathogenetic role in eczematous dermatitis. J. Clin.
Invest. 106:25-35.

13. Chan, L.S., Robinson, N., and Xu, L. 2001. Expression of interleukin- 4 in the epidermis of transgenic mice results in a pruritic inflammatory skin disease: an experimental animal model to study atopic dermatitis. J. Invest. Dermatol. 117:977-983.

14. Spergel, J.M., Mizoguchi, E., Oettgen, H., Bhan, A.K., and Geha, R.S. 1999. Roles of TH1 and TH2 cytokines in a murine model of allergic dermatitis. J. Clin. Invest. 103:1103-1111.

15. Novak, N., et al. 2003. Evidence for a differential expression of the FcepsilonRIgamma chain in dendritic cells of atopic and nonatopic donors. J. Clin. Invest. 111:1047-1056. doi:10.1172/JCI200315932.

16. Laouini, D., et al. 2003. IL-10 is critical for Th2 responses in a murine model of allergic dermatitis. J. Clin. Invest. 112:1058-1066. doi:10.1172/ JCI200318246.

17. Ong, P.Y., et al. 2002. Endogenous antimicrobial peptides and skin infections in atopic dermatitis. $N$. 
Engl. J. Med. 347:1151-1160.

18. Nomura, I., et al. 2003. Cytokine milieu of atopic dermatitis, as compared to psoriasis, skin prevents induction of innate immune response genes. J. Immunol. 171:3262-3269.

19. Sator, P.G., Schmidt, J.B., and Honigsmann, H. 2003. Comparison of epidermal hydration and skin surface lipids in healthy individuals and in patients with atopic dermatitis. J. Am. Acad. Dermatol. 48:352-358.

20. Cookson, W.O., and Moffatt, M.F. 2002. The genetics of atopic dermatitis. Curr. Opin. Allergy Clin. Immunol. 2:383-387.

21. Liu, X., et al. 2003. Associations between total serum IgE levels and the 6 potentially functional variants within the genes IL4, IL13, and IL4RA in German children: the German Multicenter Atopy Study. J. Allergy Clin. Immunol. 112:382-388.

22. Kato, A., et al. 2003. Association of SPINK5 gene polymorphisms with atopic dermatitis in the Japanese population. Br. J. Dermatol. 148:665-669.

23. Kabesch, M., et al. 2003. Association between polymorphisms in caspase recruitment domain containing protein 15 and allergy in two German popula- tions. J. Allergy Clin. Immunol. 111:813-817.

24. Leung, D.Y. 2003. Infection in atopic dermatitis. Curr. Opin. Pediatr. 15:399-404.

25. Leung, D.Y., et al. 1993. Presence of IgE antibodies to staphylococcal exotoxins on the skin of patients with atopic dermatitis. Evidence for a new group of allergens. J. Clin. Invest. 92:1374-1380.

26. Chamlin, S.L., et al. 2002. Ceramide-dominant barrier repair lipids alleviate childhood atopic dermatitis: changes in barrier function provide a sensitive indicator of disease activity. J. Am. Acad. Dermatol. 47:198-208.

27. Berth-Jones, J., et al. 2003. Twice weekly fluticasone propionate added to emollient maintenance treatment to reduce risk of relapse in atopic dermatitis: randomised, double blind, parallel group study. $B M J$. 326:1367.

28. Kapp, A., et al. 2002. Long-term management of atopic dermatitis in infants with topical pimecrolimus, a nonsteroid anti-inflammatory drug. J. Allergy Clin. Immunol. 110:277-284.

29. Boguniewicz, M., Eichenfield, L.F., and Hultsch, T. 2003. Current management of atopic dermatitis and interruption of the atopic march. J. Allergy Clin. Immunol. 112:S140-S150.

30. Rappersberger, K., et al. 2002. Pimecrolimus identifies a common genomic anti-inflammatory profile, is clinically highly effective in psoriasis and is well tolerated. J. Invest. Dermatol. 119:876-887.

31. Leung, D.Y., et al. 2003. Effect of anti-IgE therapy in patients with peanut allergy. N. Engl. J. Med. 348:986-993.

32. Kalliomaki, M., Salminen, S., Poussa, T., Arvilommi, H., and Isolauri, E. 2003. Probiotics and prevention of atopic disease: 4-year follow-up of a randomised placebo-controlled trial. Lancet. 361:1869-1871.

33. Dimitroff, C.J., Kupper, T.S., and Sackstein, R. 2003 Prevention of leukocyte migration to inflamed skin with a novel fluorosugar modifier of cutaneous lymphocyte-associated antigen. J. Clin. Invest. 112:1008-1018. doi:10.1172/JCI200319220.

34. Kupper, T.S. 2003. Immunologic targets in psoriasis. N. Engl. J. Med. 349:1987-1990.

35. Leung, D.Y. 2000. Atopic dermatitis: new insights and opportunities for therapeutic intervention. J. Allergy Clin. Immunol. 105:860-876. 